\title{
Photomechanical Effects in Azo-Polymers Studied by Neutron Reflectometry
}

\author{
Kevin G. Yager, ${ }^{1}$ Oleh M. Tanchak, ${ }^{1}$ Chris Godbout, ${ }^{1}$ \\ Helmut Fritzsche, ${ }^{2}$ Christopher J. Barrett ${ }^{1}$ * \\ ${ }^{1}$ Department of Chemistry, McGill University, Montreal, QC, Canada, H3A 2K6. \\ ${ }^{2}$ Canadian Neutron Beam Centre, National Research Council, Chalk River, ON, Canada, \\ K0J $1 \mathrm{~J} 0$.
}

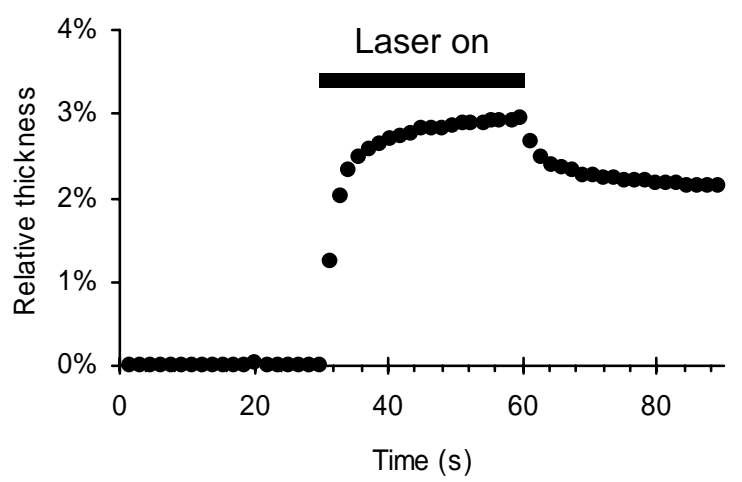

Figure S1: Relative thickness measurements, conducted using ellipsometry, measuring a distinct photo-expansion during irradiation with laser light for an azo-polymer thin film of pdr1a. While the laser is on (indicated by the bar in the figure), the material expands. Even when the laser is turned off, some amount of photo-expansion persists, indicating that there is both a reversible and irreversible component to the effect.

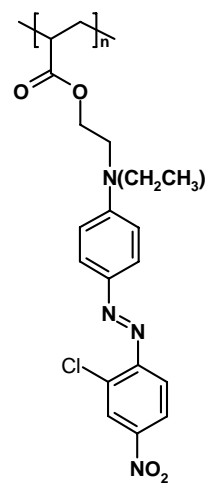

Figure S2: Chemical structure of poly(disperse red 13 acrylate) (pdr13a). 


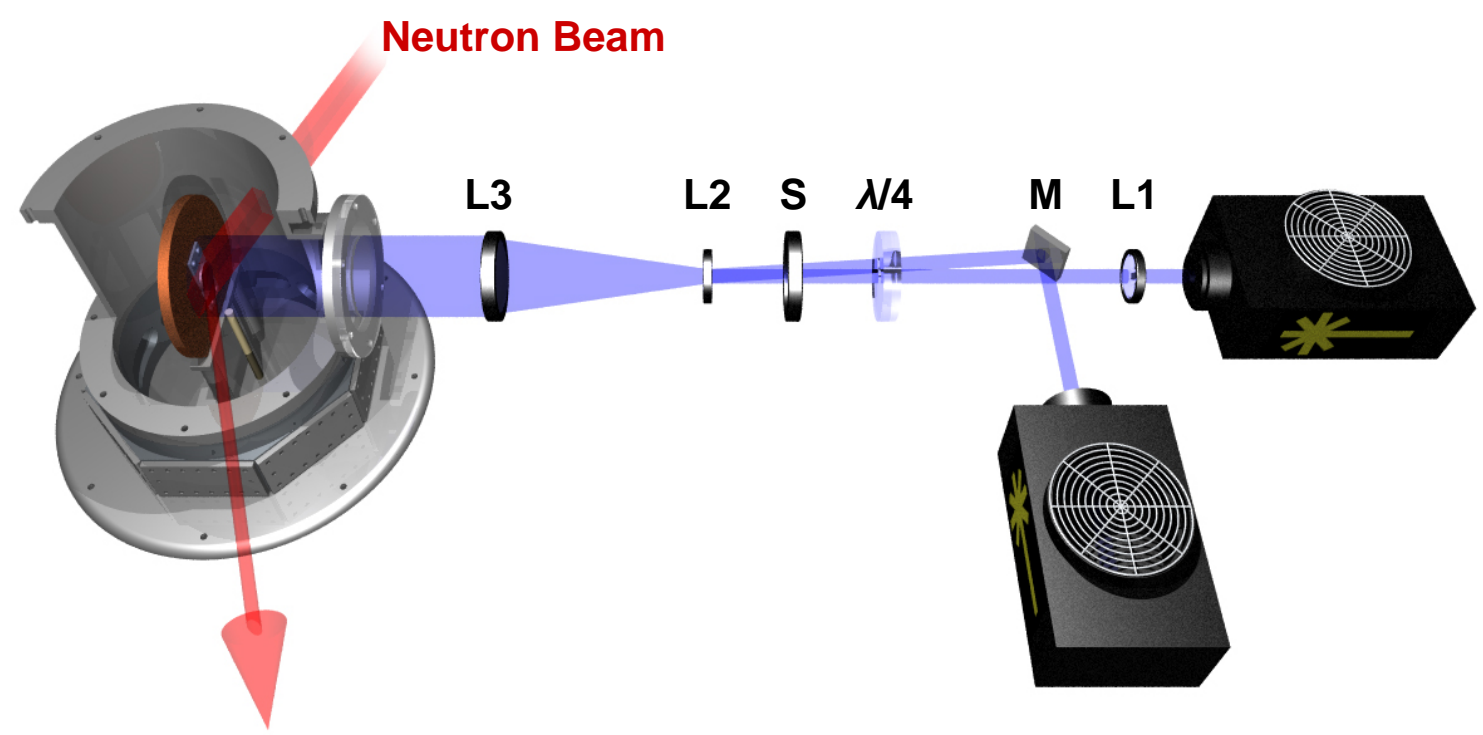

Figure S3: Experimental setup for measuring photo-physical changes in azo films using neutron reflectometry. The sample cell (left in the figure) contains a copper heating plate to which the sample wafer is attached. The aluminum enclosure is transparent to neutrons, and an optical window on the front side enables simultaneous optical irradiation. Two air-cooled $\mathrm{Ar}^{+}$lasers (on the right), tuned to $488 \mathrm{~nm}$, are used for irradiation. One laser output is adjusted with a converging lens (L1) in order to account for slight differences in divergence. The beam from the second laser is reflected off of a mirror (M), making it nearly collinear with the first beam, and passing through a concerted optical train (the angular difference in the two beams has been exaggerated in the diagram for clarity). The combined beam is converted to circular polarization using a wave plate ( $\lambda / 4)$. A diverging (L2) and converging (L3) lens are then used to expand and collimate the beam, so that it over-illuminates the sample with a uniform light intensity. The beam can be turned on and off via computer control of a shutter (S). 


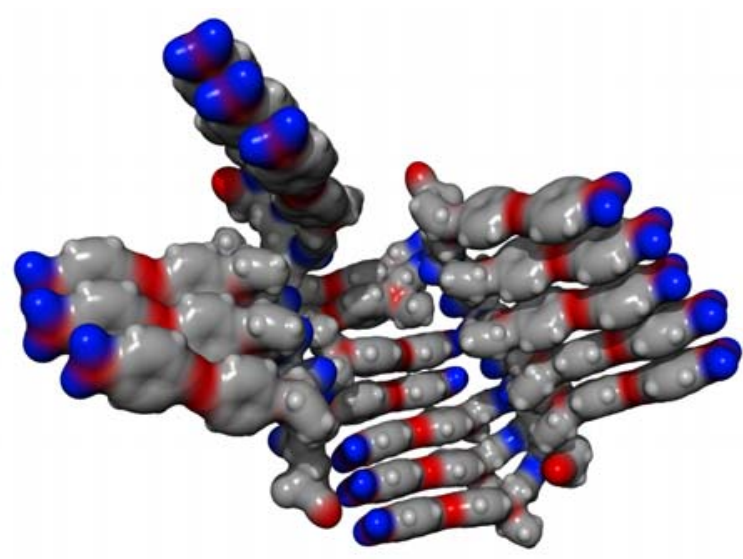

Figure S4: Molecular model of two chains (10-mers) of the azo-polymer pdr1a (generated using the Amber Force Field method from the HyperChem 6.0, Hypercube Inc. software). Carbon atoms are shown in grey, hydrogen in white, nitrogen in red, and oxygen in blue. The aromatic stacking and interdigitation of the azo chromophore side groups (seen in the center of the image) is likely the origin of the denser photo-contracted state. 